\title{
Nada es relativo. Un estudio audiovisual del posicionamiento ético de Breaking Bad
}

\author{
Ezer ez da erlatiboa. Breaking Bad-en \\ posizionamendu etikoari buruzko \\ ikus-entzunezko azterlana
}

\section{Nothing is relative. An audiovisual study of ethical positioning in Breaking Bad}

\section{Roberto Gelado Marcos 1 Javier Figuero Espadas ${ }^{2}$ Ana Lanuza Avello ${ }^{3}$}

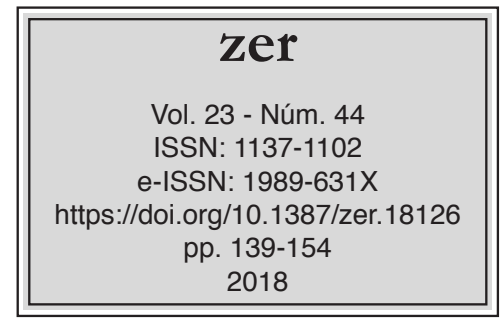

Recibido el 2 de octubre de 2017, aceptado el 9 de abril de 2018.

\section{Resumen}

La conducta ética y moral de Walter White le ha convertido en uno de los personajes de ficción que ha levantado mayor debate entre críticos, académicos y a nivel popular. En este artículo se indaga si esa fascinación sobre el antihéroe que provoca un posicionamiento moral acerca de su proceder, existe también al otro lado de la cámara. Para ello, se examinan aspectos como la escala y la angulación de los planos, la utilización de movimientos de cámara, los juegos de focos o el empleo significativo de la luz en cuatro momentos narrativos cruciales.

Palabras clave: ética; conducta; narrativa audiovisual; Breaking Bad; Vince Gilligan.

\section{Laburpena}

Walter White, bere jokabide etiko eta morala dela medio, kritikari, akademiko eta herritarren artean eztabaida handiena piztu duten fikziozko pertsonaietako bat da. Artikulu honek aztertzen du ea kameraren beste aldean ere existitzen den antiheroiaren inguruko mirespen hori, zeinak haren jokaerari buruzko posizionamendu moral bat eragiten baitu. Hainbat alderdi ikertu

\footnotetext{
Universidad CEU San Pablo, roberto.geladomarcos@ceu.es

2 Universidad CEU San Pablo, jfiguero@ceu.es

3 Universidad CEU San Pablo, ana.lanuzaavello@ceu.es
} 
dira horretarako: planoen eskala eta angeluazioa, kameraren mugimenduen erabilera, fokuen jokoak, eta argia kontakizuneko lau une garrantzitsutan erabiltzeko modu esanguratsua.

Gako-hitzak: etika; jokaera; ikus-entzunezko narratiba; Breaking Bad; Vince Gilligan.

\section{Summary}

Walter White's particular sense of Ethics has made him one of the most controversial anti-heroes for critics, academics, and spectators. This paper aims at shedding some light on whether the fascination towards White -which may lead to a moral positioning on his behaviour-runs in parallel with a similar attitude on the other end of the audio visual production. In order to do so, aspects such as the shot scales or angles will be examined, as well as camera movements, changes of focus, or the use of light in four narrative moments that are crucial.

Keywords: ethics; behaviour; audio visual narrative; Breaking Bad; Vince Gilligan. 


\section{Introducción}

Que Breaking Bad (Vince Gilligan, AMC, 2008-2013) se ha convertido en una serie de referencia apenas cuatro años después de la emisión de su último episodio es un fenómeno contrastado por sus legiones de seguidores, pero también por la prolija bibliografía de títulos más o menos académicos que sobre la serie se han publicado en los últimos tiempos.

Hay quien ha ensalzado, por ejemplo, la variada y compleja representación que hace la serie de las minorías, como apunta Howe (2014) al respecto de los retratos de ciudadanos latinos en la serie. Howe afirma que, pese a presentar arquetipos que encajan con el estereotipo habitualmente asociado a este tipo de personajes en la ficción audiovisual, especialmente americana, Breaking Bad amplía su abanico hasta llegar a perfiles más refinados, aunque delincuentes (quedan pocos que no lo son, de una manera u otra, en toda la serie, independientemente de que sean latinos o no), como el de Gus Frings. Ruiz (2015) es menos entusiasta respecto a la representación de la latinidad en Breaking Bad ya que entiende que, en el fondo, no hace sino perpetuar un trasfondo de amenaza asociado a los personajes latinos.

En un sentido similar, pero aplicado a la representación de la masculinidad, Kraynak (2015: 131) reflexiona que el arquetipo de protagonista apocado que de pronto descubre que no ha cumplido con su papel de "proveedor" es también sintomático de una psicología post-traumática con reminiscencias del 11-S. Logan (2016) también reflexiona acerca de la psicología de la serie y de sus personajes con aspectos que pueden resultar interesantes desde un abordaje ético, como por ejemplo el detectado mecanismo del autoengaño como herramienta que se les presta a algunos personajes para no verse desbordados por la realidad de sus decisiones éticas.

La construcción de personajes, central como es en casi todas las producciones audiovisuales, cobra especial relevancia en una obra tan sustentada al menos en la acción como en los arcos de transformación psicológicos y situacionales de sus personajes. Así, por ejemplo, Papet y Brillet (2014) dedican su libro sobre la serie y el perfil del antihéroe a decodificar la construcción del personaje protagonista en una metafórica clave de cambio que le emparenta con el telón de fondo químico que le da tanto el nombre de la serie como la profesión de Walt.

En tal prodigalidad de enfoques han tenido cabida también, por supuesto, reflexiones centradas en las implicaciones éticas de la serie. El planteamiento central de Breaking Bad no deja de girar, en el fondo, sobre el eterno dilema de si el fin justifica los medios; un tronco gruesísimo del que se van desgajando más y más variantes en forma de ramificaciones que parecen interrogar al espectador hasta dónde está dispuesto a seguir justificando a su protagonista. Cabe recordar, en este sentido, como señala Bruun Vaage (2010), que no es posible fascinar narrativamente al espectador sin construir una relación empática hacia su protagonista, lo cual genera en el caso de Breaking Bad, y desde su premisa misma, un dificilísimo ensamblaje de fuerzas centrífugas: el héroe se irá comprometiendo a medida que avance la serie con actos moralmente más y más condenables; $\mathrm{y}$, sin embargo, siguiendo los postulados de Bruun Vaage, sus creadores no debían descolgar nunca del todo a sus espectadores de la debida empatía profesada hacia el protagonista. Como apunta Esteban Blein (2015: 49), "es necesario acotar el tipo de respuestas emocionales que debe sentir 
el público al conocer las circunstancias del personaje, para que en efecto se pueda hablar de empatía".

El enfoque filosófico de Breaking Bad and Philosophy por Koepsell y Arp (2012) arroja interesantes discusiones, como la disquisición sobre el momento de la toma de conciencia ética de su protagonista, Walter White, acerca del decaimiento moral de sus actos desde el comienzo mismo de la serie, con el asesinato y posterior intento de deshacerse del cuerpo de Emilio, o sobre la idea misma de la posibilidad de la redención (Murphy, 2012:17). Asentada, precisamente, sobre la noción de redención está la idea de la dilución de la personalidad de los héroes trágicos que, en ese mismo libro, también aborda Glass (2012) a propósito de las dos caras de sí mismos que protagonistas como White -como le ocurre también, dice el autor, a otros como Don Draper, de Mad Men (Matthew Weiner, AMC, 2007-2015)- encuentran en su peregrinar narrativo y que centra, con su igualmente potente carga ética, buena parte de la tensión dramática de la serie.

La amplitud de su espectro narrativo da para ensayos críticos como los compilados por Pierson (2014), que abordan aspectos que van desde la política hasta la economía y la representación de minorías en la serie. Es especialmente interesante, dentro de este texto coral, el artículo de Carlo Nardi (2014) sobre la moral escurridiza de varios de los personajes de la serie. Defiende Nardi que es la propia premisa de personajes que, bien de entrada o con la propia evolución de la serie, se encuentran contaminados por una falta de ética más o menos flagrante. Eso establece una serie de relaciones que complican los veredictos y acentúan la pertinencia del análisis y las implicaciones, en materia de efectos de los medios de comunicación de masas, que puede tener la serie y su protagonista. La tradicional fascinación del espectador con el crimen (Nardi, 2014: 175) se ve elevada en Breaking Bad con el realismo de sus personajes, cuyas debilidades éticas se ven reflejadas entre unos y otros como si estuvieran en una sala de espejos.

Otros estudios abordan Breaking Bad analizando aspectos de la premisa dramática y los personajes de la serie, así como su narrativa, tratamiento visual y esquema de producción (Cobo y Hernández-Santaolalla, 2013). Entre dichos estudios, resultan especialmente relevantes aquí las reflexiones sobre las incidencias éticas del personaje de De los Ríos (2013) y Litman, que se centra especialmente en la moralidad del hecho de cocinar droga: "de todos los actos moralmente cuestionables cometidos por Walter White el más importante es su decisión de producir metanfetamina" (2013). También son muy pertinentes las deliberaciones sobre la narrativa de la cámara de Breaking Bad de Pérez de Algaba (2013).

Dentro de la colección de ensayos sobre narrativa, construcción de personajes y Ética de Blevins y Wood (2015) resultan igualmente interesantes las disquisiciones sobre la dicotomía entre ética científica y ética social de Darbeu (2015), en las que se aborda la cuestión de si el cientifismo esgrimido por White no es, en el fondo, una ética en sí mismo, un compromiso para con la ciencia que puede encontrar no solo adeptos sino una cierta justificación. En ese mismo libro también destacan las consideraciones con las que Echart y García (2015) recuperan algunas de las reflexiones esbozadas por ellos mismos sobre el carácter moral de Breaking Bad (Echart y García, 2013). La serie de Gilligan es, para ellos, ante todo una fábula moral sobre la forja de un imperio y el descenso al infierno de un hombre ordinario que, por el camino, verá cómo se ve despojado, ante todo, de sus escrúpulos. 
El orgullo es el vehículo motor de su comportamiento, como también han apuntado alguno de los otros autores referenciados en este breve apartado introductorio, aunque nuevamente la mirada vuelve a posarse esencialmente sobre el guion y la construcción de personajes, fundamentalmente el de Walter White y el que acaba siendo su contrapunto ético Jesse Pinkman, para analizar la ética del personaje planteado y no tanto el modo de plantearlo y, en última instancia, cómo esto, tanto visual como literariamente a través de parlamentos y situaciones, implica un posicionamiento moral desde la producción misma.

Hay, efectivamente, cuestiones morales en la serie que merecen un estudio detenido. La fidelidad de los espectadores iniciales de la serie parece sugerir, por ejemplo, que bastantes secundaban la moción de que el fin sí justificaba los medios si al personaje le había zarandeado tanto la vida como a Walter White, al que sin salir del primer episodio sabemos ya con cáncer de pulmón sin haber fumado un cigarrillo en su vida, con un hijo dependiente, además de un trabajo vespertino en un autolavado para pagar las facturas a las que no llega su mal pagado oficio de profesor de instituto -mérito que, además, se vislumbra muy escaso para los conocimientos que se le intuyen-. Y, si la lógica ha de entenderse en esas coordenadas, que la antedicha legión de seguidores fuese in crescendo con el paso de las temporadas no deja de parecer una reafirmación de un cierto teleologismo moral por gran parte de la audiencia que, o bien secundaba al (anti)héroe, o bien se había quedado absorta en la fascinación que el mal genera en la condición humana, como ya se sabe desde el tiempo de los griegos:

Breaking Bad juguetea con esta noción especular del abismo interior, la expresión del mal como resultado de una transformación dolorosa al tiempo que irresistible. Nos ha pasado siempre, desde la mitología griega, y nos sigue pasando en sede audiovisual: todos dimos la bienvenida al nuevo Walt y al misterioso Dr. Heisenberg. (De los Ríos, 2013).

Igualmente interesante para los propósitos de nuestra investigación es el punto de vista sobre el posicionamiento ético de Breaking Bad de Perry y Velarde (2013). Los autores aportan una serie de interesantes reflexiones sobre relativismo moral en Breaking Bad en la que se listan los ejemplos que abordan giros y parlamentos para desnudar un veredicto que se antoja incuestionable desde el principio: la doblez moral de Walter White queda de manifiesto desde sutiles esbozos como que en ningún momento se plantee revelar a su cuñado que el yonqui a quien acaba de ver en la redada del primer capítulo era alumno suyo. Si Walter, dicen Perry y Velarde, es el perfecto ejemplo del héroe nietzscheano para el que Dios ha muerto, Jesse representa el contrapunto existencialista que sobreexpone aún más la hipocresía del primero. No hay, sin embargo, menciones que superen lo meramente argumental, que para Perry y Velarde suponen más bien un punto de partida para su debate ético que, en el fondo, nunca llega a tocar aspectos más propios de la narratología, la estética o el lenguaje audiovisual.

Las implicaciones que, desde un punto de vista de los efectos de la comunicación de masas, tienen estas disquisiciones éticas son evidentes, pero tampoco son 
novedosas en el mundo de la creación audiovisual. Este temor a los efectos perjudiciales de la proyección de arquetipos tan fascinantes como nocivos para la vida en sociedad no es nada nuevo. En los años 30 del siglo pasado, en plena efervescencia de los primeros estudios de efectos y con la Academia enfrascada en el paradigma del impacto directo, las investigaciones financiadas por la fundación Payne y los estudios de Ernest Burguess para la Chicago Film Commission instauraron, por más que su cientifismo fuera dudoso, un "legado del miedo", como lo llamaron Lowery y DeFleur (1983), que acabaría desembocando en el Código Hays de 1934, del que únicamente sobrevivió la actual calificación por edades.

La pregunta -que podría perfectamente aplicarse a aquel debate catapultado por la proliferación de títulos de serie negra, estimulado a su vez por el reflejo que el cine hacía de la criminalidad alumbrada al albur de la Ley Volstead- es si no existe solo una mirada fascinada desde el otro lado de la pantalla, sino también desde detrás de las cámaras, al proyectar este tipo de arquetipos. Conviene recordar en este punto que la mirada del director es un concepto amplio que no solo se centra en la posición de cámara, sino que podría contemplar otros muchos ítems, que conforman igualmente el acto comunicativo del director en el film. Ninguno de ellos, sin embargo, tiene el carácter enunciador de la cámara ni proporciona una dimensión moral tan poderosa en la configuración de la película (Del Valle Morilla, 2017).

No han sido pocos los foros de debate en los que académicos de la Cinematografía de la Ética y otras tantas disciplinas interpeladas por la vigorosa voz de esta serie reprobaban encendidamente tal o cual comportamiento de unos y otros personajes de la serie - en el fondo Walter es solo el catalizador, pero el dilema de si el fin justifica los medios acaba salpicando a todos los personajes de una u otra manera-. En medio de estas refriegas dialécticas, resultaba absolutamente cabal preguntarse si, en el fondo, quien se empleaba con tanto ardor no cuestionaba, más que al personaje de cartón, a su creador de carne y hueso.

En este entorno de duda nace esta investigación, deseosa de arrojar algo de luz a la pregunta de si hay, realmente, más fascinación o condena desde la óptica de quien concibió esta serie, estos personajes, y estas situaciones. Dado lo gigantesco de la empresa, se ha optado por delimitar la relación entre la cámara y uno solo de los personajes, Walter White, por ser éste el hilo vehicular de prácticamente todos los demás personajes y sus respectivos designios; aunque no es ni mucho menos desdeñable abrir el camino para el análisis futuro, desde un punto de vista del posicionamiento ético desde la producción, de otros personajes y sus respectivas situaciones.

Emplearemos para nuestro análisis una muestra abarcable y circunscrita a cuatro momentos clave para el estudio de la perspectiva ética del protagonista. El objetivo de la investigación se centrará en abordar si Vince Gilligan, como creador de la serie, se posiciona moralmente o no respecto a los actos cometidos por Walter White en varios momentos del relato. La forma de hacerlo será a través del análisis de tres aspectos esenciales de la morfología y semántica cinematográfica: la planificación, el montaje y la puesta en escena. Al hablar de cinematografía nos referiremos a esa imagen que -recogiendo las ideas de Marcel Martin- Palazón explica que "podemos situar en tres diferentes niveles de realidad": la realidad 
material con valor figurativo, la realidad estética con valor afectivo y la realidad intelectual con valor significativo. (1998:19-23)

Respecto al primer aspecto, se ha tenido en cuenta la capacidad persuasiva de sus distintos elementos a la hora de determinar, desde el punto de vista audiovisual, las intenciones narrativas de los creadores y, por ende, de su posicionamiento o no respecto a los personajes y situaciones relatadas. Dentro de la planificación, se han considerado dos categorías esenciales: el tamaño del plano, que Stadler y McWilliam (2009:35) consideran el aspecto más crucial del encuadre y la composición (algo que Aumont [2004:229], parafraseando a Hitchcock, también sostiene), y la angulación, por cuanto proporciona matices igualmente interesantes respecto al posicionamiento ético (desde el carácter intimidatorio del plano nadir [Kroon, 2014:60] hasta la vulnerabilidad del picado [Kawin, 1992:209]). El enfoque y desenfoque como mecanismo narrativo que proporciona un mayor sentido al contendido del plano (Canet y Prósper, 2009: 328) es otra categoría interesante y, además, recurrente en el estilo audiovisual de la serie.

La importancia del montaje quedó definida por el propio André Bazin (2006: 95) quien sostiene que, al analizar la realidad, es el montaje el que atribuye un único sentido al acontecimiento dramático y se opone esencialmente y por naturaleza a la expresión de ambigüedad. Sangro (2011: 104) coincide en que esa concreción será posible gracias a la creación de una columna vertebral de la narración en el montaje. Respecto a la puesta en escena, término con una evidente ascendencia teatral pero con consideraciones específicas cuando se traslada a la esfera cinematográfica, como bien apuntan Bordwell et al. (1985:2 47), se ha adoptado la inclusiva acepción de Gibbs (2013), que entiende como tal todo cuanto esté contenido en el cuadro y la manera de disponerlo (aquí añadiremos, por tanto, todo lo no observado anteriormente en la planificación y el montaje: movimientos de cámara, iluminación, música, caracterización y decorados).

Estos apartados se analizarán para comprobar si proporcionan un posicionamiento neutro, favorable o contrario al personaje de Walter White, quien se va comprometiendo con actos crecientemente cuestionables desde una perspectiva ética. A partir del análisis audiovisual de estas categorías se tratará de contrastar o refutar la hipótesis principal de esta investigación.

\section{1. ¿Un posicionamiento relativista?}

Pese a que, como acabamos de ver, la enorme afluencia de reflexiones teóricas sobre las contribuciones de Breaking Bad a la cultura y el imaginario popular abordan también cuestiones éticas, parece aún preciso ahondar en las deliberaciones sobre si los creadores de la serie sugieren la condena del arquetipo del antihéroe propuesto, o bien se limitan a manifestar su fascinación por el mismo.

En una entrevista, el creador de la serie de Breaking Bad, Vince Gilligan, habla de uno de los aspectos más interesantes planteados en la serie: las consecuencias que se derivan de las acciones de los personajes, consecuencias que conectan con la perenne cuestión de la libertad humana. 
Quiero que los actos de los personajes de Breaking Bad tengan consecuencias siempre. Me imagino que es algo que en sí mismo constituye una reacción a años y años de ver televisión, a años y años de ver series en las que a un personaje le ocurre algo que le cambia la vida -matar a alguien, resultar herido- y a la semana siguiente te lo encuentras básicamente de vuelta en la normalidad, sin que se aprecien en él repercusiones emocionales de los acontecimientos que ha vivido. (Gilligan, 2013)

Walter White asesina o manda matar a cerca de 30 personas a lo largo de Breaking $\mathrm{Bad}$. En este artículo analizaremos cuatro de los crímenes más representativos en el arco dramático de caída de White, con el fin de estudiar cómo se posiciona la cámara en esos momentos y cómo refleja narrativa y visualmente la moralidad del personaje.

Los asesinatos escogidos son los de Krazy-8 (traficante distribuidor de metanfetamina), Jane Margolis (novia de Jesse Pinkman), los dos matones que acabaron con Combo (amigo de Jesse), y Mike Ehrmantraut (compinche de Gus Fring y socio de Saul Goodman). Walter White acaba con Krazy-8 en el tercer capítulo de la primera temporada (... and the Bag's in the River ${ }^{4}$ ), y la muerte de Jane Margolis tiene lugar en el final del duodécimo capítulo de la segunda temporada (Phoenix). Más adelante, Walter mata a sangre fría a los traficantes rivales que asesinaron a Combo, en el penúltimo capítulo de la tercera temporada (Half Measures) y Mike Ehrmantraut recibe un disparo a bocajarro de Walter en el séptimo episodio de la quinta temporada (Say My Name). Veamos ahora cómo se posiciona la cámara y la narración ante el proceder del antihéroe.

\subsection{La muerte de Krazy-8}

El acelerado aterrizaje de Walter White en el mundo del hampa se complica con el díptico de episodios posteriores al piloto con unas implicaciones morales mucho más espinosas. Si en el primero Gilligan había centrado todos sus esfuerzos en presentar a un personaje con la fortuna profundamente en su contra hasta extremos difíciles de sospechar, hasta el punto de que costaría entender su reacción contra la más que segura muerte a manos de dos narcotraficantes de poca monta como algo más que una desesperada defensa propia, el panorama se oscurece cuando Walter descubre que uno de sus dos agresores sigue vivo.

Durante casi dos horas de metraje el protagonista de la serie busca motivos para deshacerse de alguien que no solo ha intentado matarle, sino que a buen seguro volverá a intentarlo de ser liberado, pero no consigue ir más allá del mero asesinato preventivo como justificación. La cosa se complica aún más cuando Walter y Krazy-8 entran en territorio emocional en sus conversaciones y él le confiesa lo que aún no ha dicho a nadie, ni siquiera a su socio Jesse: que sufre cáncer. El detonante que le empujará, finalmente, a dar muerte a su rehén será el descubrimiento de que éste se

\footnotetext{
${ }^{4}$ El título del tercer capítulo finalizaba la incompleta oración del segundo, The Cat's in the Bag... La frase reproduce uno de los parlamentos de Chantaje en Broadway (Alexander Mackendrick, 1957), con el que uno de los personajes da a entender que ya se ha encargado de solucionar un asunto por el que no hay que preocuparse más y que, a su vez, parafrasea una forma relativamente antigua de paliar la sobrepoblación de gatos en algunas zonas con una eutanasia poco escrupulosa.
} 
ha aprovechado de uno de sus desvanecimientos para quedarse con un trozo de plato roto con el que, seguramente, piensa asesinar a Walter en cuanto le suelte.

Y, pese a que también podría entenderse este asesinato como defensa propia, la dirección de Adam Bernstein sobre el libreto de Vince Gilligan parecen sugerir que para ninguno de los dos son equiparables. Quizá el plano más revelador de este posicionamiento condenatorio es el que ejerce como bisagra entre el descubrimiento de Walter en la cocina y su regreso al sótano.

La cámara le encuadra entonces con un contrapicado a contraluz que no solo ensalza el poder de su figura, sino que también la ensombrece completamente, como si aquello fuese el preludio de un descenso literal en la escala de valores de un Walter que, como todo héroe trágico, está a punto de dar un paso sin vuelta atrás que le condenará a una espiral de actos cada vez más deleznables. El plano no solo se sostiene unos segundos sin que aparentemente pase nada, lo cual no hace sino reafirmar esta voluntad de Bernstein y Gilligan de dejar claro qué piensan sobre lo que su personaje está a punto de cometer, sino que además se entretiene algo más en un primerísimo primer plano de los pies de Walter que enfatiza el antedicho descenso y que acabará aterrizando, panorámica vertical mediante, en la atribulada cara del protagonista.

En la conversación que sigue a la llegada de Walter, Bernstein y Gilligan aprovechan la diferencia de altura para matizar la dinámica plano-contraplano con un juego de picados y contrapicados que ha de recordar al espectador quién ha de tomar la trascendental decisión que se avecina. Esta misma idea de quién tiene la capacidad de decidir vuelve a ponerse de manifiesto a través del juego de enfoque del subsiguiente plano a dos: se elimina el montaje para sostener la tensión con los dos personajes en el mismo cuadro; pero solo uno de ellos está enfocado, Walter, cómo no, pues la decisión final estará solo en su mano. El mismo plano a dos, con una escala aún más corta y, por consiguiente, tensa emocionalmente, presentará el mismo recurso de enfoque añadiendo el efecto del contrapicado para subrayar de nuevo que solo Walter tiene el poder de hacer algo en aquella situación.

Podría entenderse el plano detalle del bolsillo en el que Krazy-8 guarda su improvisada arma como la coartada que Gilligan y Bernstein le brindan a su protagonista para que ejecute al rehén; pero el inmediato retorno al juego de enfoques cuando Walter empieza a intentar asfixiarle parece sugerir un descarte de esta teoría. El montaje se vuelve más frenético y los planos a dos se combinan con planoscontraplanos. Pero lo más importante y revelador es que, desde que Walter decide que no hay marcha atrás y que el asesinato es la única opción posible, Bernstein y Gilligan le emborronan constantemente con el desenfocado, como si aquel fuera un paso irreversible en su autodestrucción moral. El larguísimo plano, en duración y en tiro, con el que se remata la escena no hace sino ahondar, desde la dirección, en el empequeñecimiento de un protagonista que, por primera vez, ha decidido asesinar sin que la defensa propia y la ausencia de premeditación sea un eximente tan claro como dos episodios antes en la furgoneta.

\subsection{La muerte de Jane}

En el penúltimo capítulo de la segunda temporada, con Walter ya sin pelo e instalado en el imperio de la droga, vuelve a cruzarse en su camino el fantasma de una muerte 
no tan anunciada. En su obsesivo universo del control una pieza extraña no deja fluir el engranaje como le gustaría: Jane Margolis, vecina y casera de su socio Jesse, que se acabará convirtiendo en la novia de éste y verá en la ingente cantidad de dinero que ambos pueden producir un pasaporte de lujo a una vida mejor. Al ver su recaída en los estupefacientes, Walter le corta a Jesse el suministro proporcional de beneficios y Jane reacciona chantajeando a Walter, lo que la convierte en una incómoda piedra en el camino.

Tras coincidir accidentalmente con el padre de Jane sin saber que lo es, Walter sigue los consejos de su interlocutor improvisado y va a casa de Jesse a tratar de ayudarle a reconducir sus malos hábitos. Una vez allí, y de nuevo en una escena enteramente contada desde su punto de vista como sucedía con la muerte de Krazy-8, Walter descubre, al mismo tiempo que el espectador, que Jesse y Jane han estado consumiendo heroína.

Aprovechando la diferencia de altura entre Walter y sus embriagados interlocutores sobre la cama, el director del capítulo escrito por John Shiban, Colin Bucksey, vuelve otra vez a presentar al protagonista de la serie en un conocido contrapicado, exactamente igual que cuando entró en el sótano para dar muerte a Krazy-8. Es solo, como veremos, uno de los patrones que se repiten en la recurrente idea de que, por más que su punto de vista pueda sugerir lo contrario, siempre es Walter quien tiene la última palabra a la hora de decidir si comprometerse con actos más luctuosos o no.

Aunque la muerte de Jane puede plantear, en un principio, más interrogantes morales que la de Krazy-8 por no tratarse de un asesinato a quemarropa sino de una omisión del deber de socorro, tanto Shiban como Bucksey optan por no alinearse en términos empáticos con Walter desde un primer momento. Solo en esa clave se puede entender, por ejemplo, el plano cenital (y ligeramente aberrado, tal vez anticipador de lo que habrá de suceder) en el que se ve cómo, al tratar de despertar a Jesse, Walter pone accidentalmente a Jane boca arriba. Aquel fino detalle, que además ya había sido anticipado por parlamento previamente para esquivar acusaciones de jugar con ventaja, pone de manifiesto que en realidad Walter no solo está evitando ayudar a Jane (lo cual, en términos éticos ya sería difícilmente defendible), sino que sin su presencia en la habitación aquella noche, ella nunca hubiera muerto asfixiada. Que Shiban y Bucksey hagan un aparte visual para mostrar el detalle no hace sino redundar en un retrato poco excusatorio de su protagonista.

El posterior juego de enfocados y desenfocados con el que se representa la muerte por asfixia de Jane regresa a lugares comunes ya visitados con la muerte de Krazy-8 y los perfecciona con una angulación aberrada que, de nuevo, pone de manifiesto el descontrol de la situación. Hay aquí dos planos exactamente idénticos que se intercalan en dos puntos clave del montaje: uno, cuando Jane puede aún ser salvada; y otro, cuando Walter no puede hacer nada. En ambos planos, además del encuadre aberrado, Jane copa uno de los tercios inferiores del cuadro porque es nada menos que su vida lo que está en juego; si bien en el primero está desenfocada porque no está en su mano decidir si vive o si muere. Walter está empequeñecido al lado opuesto; pero, a diferencia de su compañera de cuadro, está plenamente enfocado, lo cual no puede entenderse sino como un recordatorio de su posición prevalente a la hora de decidir los designios de la novia de Jesse. 
En el segundo plano gemelo, la situación ha cambiado: Jane ha muerto y Walter ya no puede hacer nada para remediarlo. La composición es idéntica, pero el juego de enfoques ha cambiado, no tanto porque la muerte haya podido ser ese elemento catártico que ha afinado la silueta de Jane; sino, sobre todo, porque Walter ha vuelto a quedar, como cuando asesinó a Krazy-8, tan emborronado como su propia altura moral.

El coleo de casi medio minuto sostenido casi únicamente en un primer plano del compungido rostro de Walter, solo aliviado por el breve retorno al cenital de la cama con Jane muerta y Jesse ajeno a todo, acaba siendo un colosal corolario visual que obliga al protagonista a mirarse al espejo excretor de sus repugnantes actos durante más tiempo que el que su conciencia, probablemente, elegiría.

\subsection{La muerte de los traficantes rivales}

La dualidad existencial de Jesse entre el ser real y el ser pensado, el ético, el que debería ser (evoquemos la magistral escena de la fabricación de la caja de madera frente a su esclavitud real cocinando cristal encadenado) aparece plasmada en este momento en el que el personaje quiere obrar, pero no distingue cómo, por lo que encontrará en las drogas el ímpetu para desenvolverse. El joven Pinkman no parece estar hecho para la venganza, de ahí ese aliciente de diseño para enfrentarse a sus rivales. Jesse consigue una pistola y espera en el interior de su coche a los asesinos de su amigo.

Cuando llegan los traficantes rivales, Jesse coge la pistola que oculta bajo su asiento, sale y se dirige a ellos, quienes, al verle venir, se enfrentan a él mostrando sus armas, primer tanteo de los bárbaros imponiendo sus colmillos intimidadores.

$\mathrm{El}$ acercamiento de Jesse se produce en un primer plano que nos muestra su rostro ligeramente desenfocado, enfatizando la ira y el caos que alberga el personaje, icono henchido y amenazador de una mente nublada a punto de incurrir en un crimen para el que no parece preparado. No hay nada en foco en este primer plano, donde resalta la cara de Jesse frente a un segundo término indiscutiblemente borroso.

Seguidamente, vemos a Jesse de espaldas mientras avanza. Al fondo sus oponentes, fuera de foco. El joven aún posee la iniciativa de la acción, que se disipará cuando el plano cambie el foco a los traficantes. El objetivo de Jesse -sus rivales- es ahora lúcido, un enfoque-desenfoque parece haber determinado su destino, subrayado en la siguiente toma, donde vemos a Jesse perfectamente nítido: la intención del joven parece ahora clara, precisa e inequívoca. No hay ya vuelta atrás.

Toda la escena se plantea, recurso habitual en Breaking Bad, como un duelo en el viejo Oeste, en la que no falta el plano a ras de suelo de los pies de Jesse filmados desde atrás avanzando hacia sus oponentes, que rememora las ajadas botas vaqueras de los duelos en los westerns.

Jesse se acerca a ellos lo suficiente y desenfunda, los otros hacen lo mismo, pero justo en ese momento, antes de que se produzcan los disparos, se oye derrapar a un coche, vemos un plano de la rueda de un vehículo girando veloz y aparece Walter White, que atropella violentamente a los dos matones. Tras el dislate, lo primero que vemos es un plano cerrado del brazo inmóvil de uno de los matones que sobresale bajo el coche, se abre la puerta del vehículo y aparecen los pies de Walter, que viendo que uno de los traficantes aún se mueve, se acerca a él, coge su pistola del suelo (lo 
vemos en un plano inserto) y le dispara en la cabeza a sangre fría, para inmediatamente después mirar a Jesse desafiante. Jesse está en estado de shock y sin saber reaccionar, se ha quedado petrificado. Plano medio contrapicado de Walter White, travelling de aproximación hacia su cara, y un grito a Jesse: “¡corre!”. Hay un fundido a negro, con el que concluye el episodio.

Justo antes del atropellamiento hay un momento muy significativo: Jesse, en plano medio, mira asombrado la escena y es ocultado completamente por el coche que conduce Walter White al pasar entre la cámara y Jesse. El joven es literalmente tragado por la masa negra del coche. Una vez más, Walter ha oscurecido y hecho desaparecer a su socio. Walter comete un crimen con el que salva la vida de Jesse -un colaborador necesario e imprescindible- para poder seguir con su plan de cocinar droga. El ocultamiento de Jesse por el coche puede verse como una metáfora de la manipulación que ejerce Walter White sobre él, precisamente en los momentos decisivos de la vida del joven: Walter sigue conduciendo a Jesse hacia donde él decide.

En los comentarios a esta escena, sus creadores -entre otros, el director del episodio, Adam Bernstein, y uno de los guionistas, Peter Gould-, hacen referencia a cómo la cámara lenta es una de las claves narrativas del momento en el que Jesse se acerca a sus oponentes. Y como no podía ser de otro modo, aluden al Western al afirmar que el corte de los planos del montaje final recuerda a una película de Sergio Leone. (Bernstein: 2013).

\subsection{La muerte de Mike}

La escena de la muerte de Mike está también grabada con múltiples evocaciones al género western, lo que la convierte en un curioso duelo entre el bueno (Mike) y el malo (Walter). El malo actúa a traición para acabar con su rival, empujado por un ego desmedido y un engreimiento herido. Podemos afirmar, siguiendo a Echart y García, que, una vez más, el sentimiento de orgullo prevalece sobre el remordimiento.

¿Cómo recoge la cámara ese orgullo de Walter? La escena empieza con un primer plano de unas botas que avanzan con seguridad por un polvoriento camino, durante unos instantes el objetivo sigue a las botas para luego elevarse y dejarnos ver a Mike, que se acerca a un coche que se aproxima a lo lejos. La cámara se eleva hasta un gran plano general desde el que vemos muy diminutos a los dos protagonistas en un cruce de caminos donde se produce el encuentro, en medio del paisaje arbolado y bajo un cielo azul. El espacio, como en los westerns, cobra una importancia decisiva en el planteamiento de la historia.

La cámara se posiciona desde el primer instante con el personaje cuyos pasos sigue desde el principio: es a Mike a quien vemos mientras la grúa se eleva, y es a quien sigue mientras a lo lejos se aproxima el coche de Walter, que claramente no es el protagonista en este momento de la historia.

Cuando llega Walter, se baja del coche y tiene lugar una conversación en planos cada vez más cerrados, que reflejan -con cada acercamiento- los sentimientos in crescendo de orgullo (Walter) y cólera (Mike). Con una clarividencia rotunda Mike pronuncia un discurso que ha pasado a formar parte de los célebres diálogos de Breaking Bad: 
Teníamos algo bueno, estúpido hijo de puta, teníamos a Fring, teníamos un laboratorio, teníamos amigos, y todo funcionaba con la precisión de un reloj, podías haber cerrado tu boca, cocinar y ganar más dinero del que nunca necesitarás. Era perfecto, pero no, tenías que fastidiarlo todo, tú, con tu orgullo y tu ego. Tenías que ser el gran hombre. Si hubieras hecho tu trabajo y sabido cuál era tu lugar, todos estaríamos perfectamente ahora. (Gilligan y Schnauz, 2013).

La discusión acaba con negativa de Mike a darle una lista de los presos que Walter piensa que podrían delatarle. Vemos a Mike va a su coche y sale de plano. Walter queda frente a nadie, rabioso por el desplante recibido. Finalmente, Walter da la media vuelta y decide también irse a su coche. El plano queda vacío durante uno, dos, tres segundos... De pronto Walter reaparece por donde se había ido y se encamina furioso hacia el coche de Mike.

Desde el interior de su coche, vemos cómo Mike revisa la bolsa que Walter le ha traído (esta es la excusa del encuentro). La cámara está en el asiento del copiloto, vemos la bolsa y a Mike. Repentinamente en segundo término aparece Walter junto a la ventanilla y encañona a Mike desde fuera del coche. Ni la profundidad de campo ni la ventanilla polvorienta nos permiten ver nítidamente a Walter. De esta forma, la cámara se posiciona con Mike en el momento de su muerte. Walter, en segundo término, desenfocado y poco visible, parece no obtener la aquiescencia de la mirada del realizador en este instante de perversidad. Hay una clara jerarquización que excluye al antihéroe: "esta jerarquización visual no siempre está vinculada al poder sino también a la acogida social de un personaje [como es el caso], información recogida generalmente por la distribución de los individuos dentro del encuadre, que se traduce en una determinada profundidad espacial" (Pérez de Algaba, 2013)

La cámara sale del coche para mostrar un plano de la ventanilla de Mike, y a Walter de escorzo (otra vez la realización ignora al asesino) para enseñar cómo la ventanilla queda hecha trizas por el impacto de la bala. De nuevo volvemos al interior del coche, con el mismo posicionamiento de la cámara anterior, para ver a un Mike herido por el disparo, que trata de huir de su asesino.

Mientras el coche se aleja, por primera vez aparece Walter nítido tras el disparo a través de un gran angular que deforma sutilmente la perspectiva. Walter parece sorprendido y asustado de su propia acción, mira hacia delante incrédulo, la tergiversación del objetivo ayuda a articular lo irracional de la acción, que además carece de sentido, como reconoce el propio Walter a un Mike herido de muerte: "Me acabo de dar cuenta que Lydia tiene los nombres. Se los puedo pedir a ella. Lo siento." (Gilligan y Schnauz, 2013). Mike muere mirando la serena corriente del río, de hecho, Mike le pide a Walter -cuando aquel se está disculpando por lo ocurrido-, que cierre su boca y le deje morir en paz, mirando la corriente de agua y contemplando por última vez el hermoso paisaje que le rodea.

\section{Conclusiones}

El análisis de cuatro de los momentos cumbre en el mapa narrativo de Breaking Bad confirma una percepción muy extendida: Walter White, el protagonista de la serie, 
emerge a lo largo de la serie como un antihéroe sumamente perturbador; un hombre aparentemente corriente que se ve imbuido en un proceso de vértigo y, fascinado por el mal, irá corrompiéndose progresivamente a medida que sus acciones se vuelven más y más deplorables. Sin embargo, un estudio más profundo, articulado en torno a las tres categorías propuestas en la base metodológica de este artículo, la planificación, el montaje y la puesta en escena, revelan conclusiones aún más interesantes.

$\mathrm{El}$ análisis del posicionamiento moral de la cámara ante cuatro acontecimientos clave en el descenso a los infiernos éticos de Walter White (las muertes de Krazy-8, Jane Margolis, los dos matones que acabaron con Combo, y Mike Ehrmantraut) sugiere que, efectivamente, los creadores de la serie no optaron por una presentación audiovisual aséptica de los hechos filmados. Existe un evidente énfasis en el punto de vista del protagonista -lo contrario no se concibe en ningún relato audiovisual-, pero todos los episodios narrativos sometidos a análisis sugieren un posicionamiento condenatorio de los comportamientos que van hundiendo moralmente de manera progresiva a Walter White.

Tras el análisis de la narrativa y el lenguaje audiovisual de dichos asesinatos, tales como el tamaño de plano, la angulación, los movimientos de cámara o los puntos de foco, hay motivos para afirmar que las cuatro escenas incluyen sistemáticamente trazas condenatorias hacia las acciones de Walter White y su creciente compromiso con su lado más oscuro. La cámara, en fin, toma partido respecto a la ética de las acciones de su personaje principal a través del uso de determinados recursos narrativos en momentos claves. A la luz de este análisis, así pues, se confirma que el relato audiovisual de Breaking Bad sí se posiciona moralmente en contra de los actos de su protagonista.

Esta afirmación, que cabe colegir de los resultados de nuestro análisis, introduce consideraciones muy interesantes que nos remontan a la esencia misma de las primeras reflexiones sobre el impacto de las piezas audiovisuales, también comentadas con anterioridad. Siendo innegable que la idealización por parte de ciertos sectores de la audiencia de personajes de comportamiento reprobable puede ser, hasta cierto punto, inevitable. Ello no debe desviarnos, en cualquier caso, de la responsabilidad del comunicador en la creación de contenidos, especialmente cuando, como ocurre en Breaking Bad, el resultado final no es una mera idealización del personaje; es más, no es una idealización en sentido estricto.

En la fina línea que puede empujar la creación audiovisual hacia el inhóspito lodazal del anteriormente referido legado del miedo, en el que cualquier producción que hable de temas éticamente delicados corre el riesgo de ser directamente etiquetada como apologética de conductas indeseables, no hay argumento más poderoso desde el lado de la creación que defender que hablar de ciertos temas, como en este caso la corrupción moral del hombre corriente, no significa necesariamente apoyarlos. Por eso, precisamente, los resultados que arroja el presente estudio demuestran que Vince Gilligan no solo es perfectamente consciente de la importancia de este posicionamiento ético, sino que hace de él un ejercicio absolutamente responsable. 


\section{Referencias bibliográficas}

Aumont, J. (2004). Aesthetics of Film. Austin (TX): University of Texas Press, $5^{\mathrm{a}} \mathrm{ed}$. $\left(1^{\text {a }}\right.$ ed.1983).

Bazin, A. (2006). ¿Qué es el cine? Madrid: Rialp

Bernstein, A. (2013). Half Measures. DVD. Los Ángeles: Sony Pictures.

Blevins, J. \& Wood, D. (2015). The Methods of Breaking Bad: Essays on Narrative, Character and Ethics. Jefferson (NC): McFarland.

Bordwell, D., Staiger, J. \& Thompson, K. (1985). The Classical Hollywood Cinema. Film Style \& Mode of Production to 1960. Londres: Routledge.

Gibbs, J. (2013). Mise-en-scène: Film Style and Interpretation. Nueva York: Columbia University Press.

Bruun Vaage, M. (2010). "Fiction Film and the Varieties of Empathic Engagement", Midwest Studies in Philosophy, XXXIV (pp. 158-79).

Darbeu, R. W. (2015). Scientific Ethics and Breaking Bad. En Jacob Blevins \& Dafydd Wood (Eds.), The Methods of Breaking Bad: Essays on Narrative, Character and Ethics (pp. 165-82). Jefferson (NC): McFarland.

Canet, F y Prósper, J (2009). Narrativa audiovisual. Estrategias y recursos. Madrid: Editorial Síntesis.

De Los Ríos, I. (2013). Tres objeciones de amor y una ovación desesperada. En Cobo, S. y Hernández-Santaolalla, V. (Eds.), Breaking Bad. 530 gramos (de papel) para serieadictos no rehabilitados (pp. 23-45). Madrid: Errata naturae.

Del Valle Morilla,A. M. (2017). Propuesta de un modelo de análisis cinematográfico integral y aplicación del modelo a Rear Window (La ventana indiscreta, 1954) de Alfred Hitchcock. Comunicación y Hombre, 13, 183-199.

Echart, P. \& García, A. N. (2013). Crime and Punishment: Greed, Pride and Guilt in Breaking Bad. En Alexandra Simón-López \& Heidi Yeandle (Eds.), A Critical Approach to the Apocalypse (pp. 205-217). Oxford: Inter-Disciplinary Press.

Echart, P. \& García, A. N. (2015). Round the decay / Of that colossal wreck: Pride and Guilt as Narrative Emotions. En Jacob Blevins \& Dafydd Wood (Eds.), The Methods of Breaking Bad: Essays on Narrative, Character and Ethics (pp. 78-93). Jefferson (NC): McFarland.

Esteban Blein, J. (2015) El antihéroe como protagonista en el guion épico americano contemporáneo: estrategias identificatorias y evolución heroica. Tesis Doctoral. Universidad CEU San Pablo Facultad de Humanidades y CC. de la Comunicación. Madrid.

Gilligan, V. (2013) Así hacemos Breaking Bad. En Gilligan, V. (Ed.), Breaking Bad, (530) gramos (de papel) para serieadictos no rehabilitados (pp-61-99). Madrid: Errata naturae.

Gilligan, V.\& Schanauz, T. (2013). Say My Name.DVD. Los Ángeles: Sony Pictures.

Glass, S. (2012). Better Than Human. En David R. Koepsel \& Robert Arp (eds.). Breaking Bad and Philosophy: Badder Living through Chemistry. Chicago: Open Court, pp. 91-100.

Howe, D. (2014). Not Your Average Mexican: Breaking Bad and the Destruction of Latino Stereotipes. En David Pierson (ed.). Breaking Bad: Critical Essays on the Contexts, Politics, Style, and Reception of the Television Series. Lanham: Lexington Books, pp. 87-102. 
Kawin, Bruce F. (1992). How Movies Work. Berkeley y Los Angeles (CA): University of California Press.

Kroon, R. (2014). A/V A to Z: An Encyclopedic Dictionary of Media, Entertainment and Other Audiovisual Terms. Carolina del Norte: McFarland \& Co.

Koepsel, D. R. \& Arp, R. (2012). Breaking Bad and Philosophy: Badder Living through Chemistry. Chicago: Open Court.

Kraynak, S. (2015). The Danger that Keeps Knocking. Representations of Post-9/11 Masculinity in Vince Gilligan's Breaking Bad. En Paul Petrovic (Ed.), Representing 9/11: Trauma, Ideology, and Nationalism in Literature, Film, and Television, (pp. 131-142). Londres: Rowman \& Littlefield.

Litman, G. (2013). ¿Cocinar coloca a Walt en el lado de los "malos"? En Cobo, S. y Hernández-Santaolalla, V. (Eds.), Breaking Bad. 530 gramos (de papel) para serieadictos no rehabilitados (pp 45-63). Madrid: Errata naturae.

Logan, E. (2016). Breaking Bad and Dignity: Unity and Fragmentation in the Serial Television Drama. London: Palgrave Macmillan

Nardi, C. (2014). Mediating Fictional Crime, Music, Morality and Liquid Identification. En David Pierson, (ed.). Breaking Bad: Critical Essays on the Contexts, Politics, Style, and Reception of the Television Series. Lanham: Lexington Books, pp. 173-90.

Murphy, D. J. (2012). Heisenberg's Uncertain Confession. En David R. Koepsel \& Robert Arp (eds.). Breaking Bad and Philosophy: Badder Living through Chemistry. Chicago: Open Court, pp. 15-26.

Palazón, A. (1998) Lenguaje audiovisual. Madrid: Acento Editorial.

Papet, C. \& Brillet, A. (2014). Breaking Bad, le culte de l'anti-héros. Paris: Clément.

Pérez De Algaba, C. (2013). Breaking Bad plano a plano. La cámara como constructora de significado. En Cobo, S. y Hernández-Santaolalla, V. (Eds.), Breaking Bad. 530 gramos (de papel) para serieadictos no rehabilitados (pp. 295-311). Madrid: Errata naturae.

Perry, B. \& Velarde, R. (2013). Breaking Bad's Addicting Defense of Moral Realism. Christian Research Journal, $\mathrm{n}^{\circ} 5$.

Pierson, D. P. (2014). Breaking Bad: Critical Essays on the Contexts, Politics, Style, and Reception of the Television Series. Lanham: Lexington Books.

Ruiz, J. (2015). Dark Matters: Suburban Crime Dramas, and Latinidad in the Golden Age of Cable Television. Aztlan: A Journal of Chicano Studies, Volumen 40, Número 1, Primavera 2015, 37-62.

Sangro, P. (2011). La práctica del visionado cinematográfico. Madrid: Editorial Síntesis. Stadler, J. \& McWilliam, K. (2009). Screen Media: Analysing Film and Television. Crows Nest (Australia): Allen \& Unwin.

[2] El título del tercer capítulo finalizaba la incompleta oración del segundo, The Cat's in the Bag... La frase reproduce uno de los parlamentos de Chantaje en Broadway (Alexander Mackendrick, 1957), con el que uno de los personajes da a entender que ya se ha encargado de solucionar un asunto por el que no hay que preocuparse más y que, a su vez, parafrasea una forma relativamente antigua de paliar la sobrepoblación de gatos en algunas zonas con una eutanasia poco escrupulosa. 